\title{
SIMPLE ROOT SYSTEMS AND PRESENTATIONS FOR CERTAIN COMPLEX REFLECTION GROUPS
}

\author{
JIAN-YI SHI \\ Department of Mathematics, East China \\ Normal University, Shanghai, 200062, China \\ and \\ Center for Combinatorics, Nankai \\ University, Tianjin, 300071, China
}

\begin{abstract}
We find all the inequivalent simple root systems for the complex reflection groups $G_{12}, G_{24}, G_{25}$ and $G_{26}$. Then we give all the non-congruent essential presentations of these groups by generators and relations. The methed used in the paper is applicable to any finite (complex) reflection groups.
\end{abstract}

\section{Introduction.}

Shephard and Todd classified all the finite complex reflection groups in paper [5]. Later Cohen gave a more systematic description for these groups in terms of root systems $(R, f)$ and root graphs in [2], in particular, he defined a simple root system $(B, w)$ for the root system of such a group, which is analogous to the corresponding concept for a Coxeter group. In general, for a given finite complex reflection group $G$, a root system $(R, f)$ is essentially unique but a simple root system $(B, w)$ for $(R, f)$ is not (even up to $G$-action), e.g., when $G=G_{33}, G_{34}$ (see [1, Table 4]). One can define an equivalence relation on the set of simple root systems for $(R, f)$ (see 1.7). A natural question is to ask

Problem A. How many inequivalent simple root systems are there in total for any irreducible finite complex reflection group $G$ ?

Key words and phrases. complex reflection groups, simple root systems, congruence presentations.

Supported by the 973 Project of MST of China, the NSF of China, the SF of the Univ. Doctoral Program of ME of China and the Shanghai Priority Academic Discipline 
It is well known that any Coxeter group can be presented by generators and relations. A finite complex reflection group $G$ can also be presented in a similar way (see [2]). But such a presentation is not unique for $G$ in general. Different presentations of $G$ may reveal various different properties of $G$ (see [6] for example). Then it is worth to define a congruence relation among the presentations of $G$ (see 1.9) and then to ask

Problem B. How many non-congruent presentations are there for any irreducible finite complex reflection group $G$ ?

In the present paper, we solve these problems just for the finite primitive complex reflection groups $G=G_{12}, G_{24}, G_{25}, G_{26}$ (in the notations of [5]). The other finite primitive complex reflection groups except $G_{34}$ will be dealt with subsequently in two papers by my graduate students Li Wang and Peng Zeng (see [8, 9]). We choose to deal with these four groups as they represent four different cases. Among these groups, $G_{12}$ is the only group the number of whose generating reflections is not equal to the dimension of the space it acts. The group $G_{24}$ is generated by three reflections of order $2, G_{25}$ is generated by three reflections of order 3 , while $G_{26}$ is generated by three reflections of different orders: one of order 2 and two of order 3. Our method in dealing with Problem A is based on the knowledge of the action of $G$ on its root system. Then the results so obtained, together with a known presentation of $G$, will be used in dealing with Problem B. We shall further show that the reduced forms for the presentations of the groups obtained in the paper are all essential (see 7.1 and Theorem 7.3). It is a relatively easy task if one is only content with getting the non-congruent essential presentations for a given finite complex reflection group. But some of such presentations may have very complicated form and hence is not applicable in practice. So in the present paper we are trying to find the forms of the non-congruent essential presentations as simple as possible. Then it becomes quite subtle in finding such forms and even more subtle in proving that they are indeed non-congruent and essential. The methods used in the paper are applicable to any other finite (complex) reflection group.

The contents are organized as follows. Section 1 is served as preliminaries, some definitions and results are collected there. Then we give all the inequivalent simple root systems for the group $G_{12}$ in Section 2, and give all the non-congruent presentations of $G_{12}$ in Section 3. We do the same for the groups $G_{24}, G_{25}, G_{26}$ in Sections 4, 5, 6, respectively. Finally we show in Section 7 that the reduced forms for all the presentations of the groups obtained in the paper are essential. 


\section{$\S 1$ Preliminaries.}

We collect some definitions and results concerning irreducible finite reflection groups, where 1.1-1.5 follow from Cohen's paper [2] except for the definition of a simple root system which follows from [3].

1.1. Let $V$ be a complex vector space of dimension $n$. A reflection on $V$ is a linear transformation on $V$ of finite order with exactly $n-1$ eigenvalues equal to 1 . A reflection group $G$ on $V$ is a finite group generated by reflections on $V$. The group $G$ is reducible if it is a direct product of two proper reflection subgroups and irreducible otherwise. The action of $G$ on $V$ is said to be irreducible if $V$ has no nonzero proper $G$-invariant subspace. In the present paper we shall always assume that $G$ is irreducible and acts irreducibly on $V$. A reflection group $G$ on $V$ is called a real group or a Coxeter group if there is a $G$-invariant $\mathbb{R}$-subspace $V_{0}$ of $V$ such that the canonical map $\mathbb{C} \otimes_{\mathbb{R}} V_{0} \rightarrow V$ is bijective. If this is not the case, $G$ will be called complex. (Note that, according to this definition, a real reflection group is not complex.)

Since $G$ is finite, there exists a unitary inner product (, ) on $V$ invariant under $G$. From now on we assume that such an inner product is fixed.

1.2. A root of a reflection on $V$ is an eigenvector corresponding to the unique nontrivial eigenvalue of the reflection. A root of $G$ is a root of a reflection in $G$.

Let $s$ be a reflection on $V$ of order $d>1$. There is a vector $a \in V$ of length 1 and a primitive $d$-th root $\zeta$ of unity such that $s=s_{a, \zeta}$, where $s_{a, \zeta}$ is defined by

$$
s_{a, \zeta}(v)=v+(\zeta-1)(v, a) a \quad \text { for all } v \in V
$$

We also write $s_{a, d}$ for $s_{a, \zeta}$ if $\zeta=e^{2 \pi i / d}$. In this case, we often write $s_{a, d}$ simply by $s_{a}$ if $d$ is clear from the context.

For each $v \in V$ define $o_{G}(v)$ to be the order of the (necessarily cyclic) group that consists of the identity and the reflections in $G$ which have $v$ as a root. (This group is $G_{W}=\{g \in G \mid g u=u$ for all $u \in W\}$, where $W=v^{\perp}$.) Thus $o_{G}(v)>1$ if and only if $v$ is a root of $G$. If $a$ is a root of $G$, then $o_{G}(a)$ will be called the order of $a$ (with respect to $G)$. We shall denote $o_{G}(a)$ simply by $o(a)$ when $G$ is clear from the context.

The following results can be shown easily.

Lemma 1.3. (1) $o(g v)=o(v)=o(c v)$ for all $v \in V, g \in G$ and $c \in \mathbb{C} \backslash\{0\}$.

(2) $g s_{a} g^{-1}=s_{g(a)}$ for any $g \in G$ and any root a of $G$. 
1.4. A pair $(R, f)$ is called a root system in $V$, if

(i) $R$ is a finite set of vectors $v$ spanning $V$ with $|(v, v)|=1$;

(ii) $f: R \rightarrow \mathbb{N} \backslash\{1\}$ is a map such that $s_{a, f(a)} R=R$ and $f\left(s_{a, f(a)}(b)\right)=f(b)$ for all $a, b \in R$;

(iii) the group $G$ generated by $\left\{s_{a, f(a)} \mid a \in R\right\}$ is a finite reflection group, and for all $a \in R$ and $c \in \mathbb{C}$,

$$
c a \in R \Longleftrightarrow c a \in G a
$$

The group $G$ is called the reflection group associated with the root system $(R, f)$. We have $o_{G}(a)=f(a)$ for any $a \in R$.

We shall denote a root system $(R, f)$ simply by $R$ when $f$ is clear from the context.

1.5. A simple root system is a pair $(B, w)$, where $B$ is a finite set of vectors spanning $V$ and $w$ is a map from $B$ to $\mathbb{N} \backslash\{1\}$, satisfying the following conditions:

(i) for all $a, b \in B$, we have $|(a, b)|=1 \Longleftrightarrow a=b$;

(ii) the group $G$ generated by $S=\left\{s_{a, w(a)} \mid a \in B\right\}$ is finite;

(iii) there is a root system $(R, f)$ with $R=G B$ and $f(a)=w(a)$ for all $a \in B$;

(iv) the group $\mathrm{G}$ cannot be generated by fewer than $|B|$ reflections.

We call the elements of $S$ simple reflections. We also call $(R, f)$ the root system of $G$ generated by $B$, and $B$ a simple root system for $R$ (or for $G$ ).

Note that we do not require $B$ to be linearly independent. If $B$ is linearly independent, then condition (iv) holds automatically.

The above definition of a simple system is considerably weaker than the usual definition for Coxeter groups; in particular, it is not always true that if $B_{1}$ and $B_{2}$ are simple root systems for the same root system $R$ then there is an element $g \in G$ with $g B_{1}=B_{2}$.

By Lemma 1.3 we see that if $\alpha \in B$ and $\beta \in \mathbb{C} \alpha \cap R$, then $B^{\prime}=(B \backslash\{\alpha\}) \cup\{\beta\}$ also forms a simple root system for $R$.

1.6. Let $B$ (resp. $B^{\prime}$ ) be a subset of $V$ and $w: B \longrightarrow \mathbb{N} \backslash\{1\}$ (resp. $w^{\prime}: B^{\prime} \longrightarrow \mathbb{N} \backslash\{1\}$ ) be a function. Let $G$ (resp. $G^{\prime}$ ) be the reflection group generated by $\left\{s_{\alpha, w(\alpha)} \mid \alpha \in B\right\}$ (resp. $\left.\left\{s_{\alpha, w^{\prime}(\alpha)} \mid \alpha \in B^{\prime}\right\}\right)$. If there exists a bijection $\phi: B \longrightarrow B^{\prime}$ such that $w(\alpha)=w^{\prime}(\phi(\alpha))$ and $(\alpha, \beta)=(\phi(\alpha), \phi(\beta))$ for any $\alpha, \beta \in B$. Then by the theory of linear algebra, we see that the assignment $s_{\alpha, w(\alpha)} \mapsto s_{\phi(\alpha), w^{\prime}(\phi(\alpha))}$ determines a group isomorphism from $G$ to $G^{\prime}$. 1.7. Let $(R, f)$ be a root system with $G$ the associated reflection group and $B$ a simple root system for $R$. Then it is known that as a root system for $G, R$ is determined by $G$ 
up to scalar factors (see $[3,1.9]$ ). However, a simple root system $B$ for $R$ is not uniquely determined by $G$. Two simple root systems $(B, w)$ and $\left(B^{\prime}, w^{\prime}\right)$ for $(R, f)$ are equivalent, written $B \sim B^{\prime}$, if there exists a bijection $\phi: B \longrightarrow B^{\prime}$ such that for any $\alpha, \beta \in B$,

(1) $w(\alpha)=w^{\prime}(\phi(\alpha))$ and,

(2) $\left\langle s_{\alpha, w(\alpha)}, s_{\beta, w(\beta)}\right\rangle \cong\left\langle s_{\phi(\alpha), w^{\prime}(\phi(\alpha))}, s_{\phi(\beta), w^{\prime}(\phi(\beta))}\right\rangle$, where the notation $\langle s, t\rangle$ stands for the group generated by $s, t$.

In particular, two simple root systems $B, B^{\prime}$ for $R$ are equivalent if one of the following cases occurs:

(a) $g B=B^{\prime}$ for some $g \in G$;

(b) Condition (2) is replaced by condition $\left(2^{\prime}\right)$ below in the above definition of $B \sim B^{\prime}$ :

$\left(2^{\prime}\right) \quad|(\alpha, \beta)|=|(\phi(\alpha), \phi(\beta))|$ for any $\alpha, \beta \in B$.

In general, not all the simple root systems are equivalent for a given reflection group. So it is natural to ask Problem A in Introduction.

It is known that at least one simple root system has been found for any irreducible finite reflection group $G$ (see [7]). We have the following criterion for a subset of a root system to be a simple root system.

Proposition 1.8. Let $G$ be a finite reflection group with $(R, f)$ the associated root system and $B$ a simple root system for $R$. Let $B^{\prime}$ be a subset of $R$ and let $G_{B^{\prime}}$ be the group generated by the reflections $s_{\alpha, f(\alpha)}, \alpha \in B^{\prime}$.

(1) $B^{\prime}$ forms a simple root system for $G$ if and only if $G_{B^{\prime}} B^{\prime}=R$ and $\left|B^{\prime}\right|=|B|$.

(2) If $B \subseteq G_{B^{\prime}} B^{\prime}$ then $G_{B^{\prime}} B^{\prime}=R$.

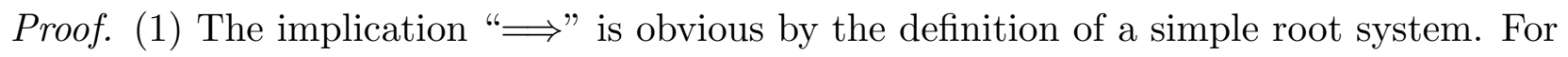
the reversing implication, we need only check conditions (i) and (ii) in 1.5. Since $G_{B^{\prime}} B^{\prime}=R$, we have $G_{B^{\prime}}=G$. So (ii) follows by the assumption that $G$ is finite. Since $(R, f)$ is a root system, any vector $v$ in $R$ (in particular, when $v$ is in $B^{\prime}$ ) satisfies $|(v, v)|=1$ and hence $\left|\left(v, v^{\prime}\right)\right| \neq 1$ for any non-proportional $v, v^{\prime} \in R$. So to show that $\left(B^{\prime}, w^{\prime}\right)$ satisfies (i), it is enough to show that $B^{\prime}$ does not contain two proportional vectors $\alpha, \beta$. For otherwise, the reflections with respect to the vectors in the proper subset $B^{\prime} \backslash\{\beta\}$ of $B^{\prime}$ would generate the group $G$, contradicting the assumptions that $\left|B^{\prime}\right|=|B|$ and that $B$ is a simple root system for $G$.

(2) Any reflection in the group $G_{B^{\prime}}$ has the form $s_{\alpha}$ with $\alpha \in G_{B^{\prime}} B^{\prime}$. Since $B \subseteq G_{B^{\prime}} B^{\prime}$ and $G=\left\langle s_{\beta} \mid \beta \in B\right\rangle$, we have $G_{B^{\prime}} \supseteq G$ and hence $G_{B^{\prime}}$ contains all the reflections $s_{\beta}$, 
$\beta \in R$. This implies the inclusion $R \subseteq G_{B^{\prime}} B^{\prime}$ and hence $G_{B^{\prime}} B^{\prime}=R$.

1.9. Given a reflection group $G$, a presentation of $G$ by generators and relations (or $a$ presentation in short) is by definition a pair $(S, \mathcal{P})$, where

(1) $S$ is a finite generator set for $G$ which consists of reflections, and $S$ has minimal cardinality with this property.

(2) $\mathcal{P}$ is a finite set of relations on $S$, and any other relation on $S$ is a consequence of the relations in $\mathcal{P}$.

Two presentations $(S, \mathcal{P})$ and $\left(S^{\prime}, \mathcal{P}^{\prime}\right)$ for $G$ are congruent, if there exists a bijection $\eta: S \longrightarrow S^{\prime}$ such that for any $s, t \in S$,

$(*)\langle s, t\rangle \cong\langle\eta(s), \eta(t)\rangle$.

In this case, we see by taking $s=t$ that the order $o(s)$ of $r$ is equal to the order $o(\eta(r))$ of $\eta(r)$ for any $r \in S$.

If there does not exist such a bijection $\eta$, then we say that they are non-congruent.

For any reflections $s, t$ in $G$, it is known (see, for example, Koster [4]) that there exists a positive integer $k$ such that $s t s \ldots=t s t \ldots$ ( $k$ factors on each side). Denote by $n(s, t)$ the smallest such $k$. It is easily seen that when $o(s)=o(t)=2$, condition $(*)$ is equivalent to condition

$(* *) \quad n(s, t)=n(\eta(s), \eta(t))$.

However, when either $o(s), o(t)>2$ or $\max \{o(s), o(t)\}=5$, one can show that neither of $(*)$ and $(* *)$ implies the other in general by checking all the cases where a complex reflection group is irreducible and is generated by exactly two reflections.

1.10. Given a simple root system $(B, w)$ for $G$, say $B=\left\{\alpha_{1}, \ldots, \alpha_{r}\right\}$, denote by $\mathbf{n}(B)$ the $\left(\begin{array}{l}r \\ 2\end{array}\right)$-tuple $\left(X_{12}, X_{13}, \ldots, X_{1 r}, X_{23}, X_{24}, \ldots, X_{2 r}, \ldots, X_{r-1, r}\right)$ with $\left(\begin{array}{l}r \\ 2\end{array}\right)=\frac{r(r+1)}{2}$, where $X_{i j}=$ $\left\langle s_{\alpha_{i}, w\left(\alpha_{i}\right)}, s_{\alpha_{j}, w\left(\alpha_{j}\right)}\right\rangle$ for $1 \leqslant i<j \leqslant r$. In particular, when $w$ is the constant function 2 , we can replace the group $X_{i j}$ by the number $n\left(s_{\alpha_{i}, 2}, s_{\alpha_{j}, 2}\right)$ for any $i, j$ in the definition of $\mathbf{n}(B)$. This is because in that case, $X_{i j}$ becomes a dihedral group which is entirely determined by the number $n\left(s_{\alpha_{i}, 2}, s_{\alpha_{j}, 2}\right)$.

1.11. For each presentation $(S, \mathcal{P})$ for $G$, there exists a simple root system $\left(B_{S}, w_{S}\right)$ for $G$ (called an associated simple root system of $(S, \mathcal{P})$ ) such that each element of $S$ has the form $s_{\alpha, w_{S}(\alpha)}^{k_{S}(\alpha)}$ for some $\alpha \in B_{S}$ and some $k_{S}(\alpha) \in \mathbb{N}$ with $k_{S}(\alpha)$ coprime to $w_{S}(\alpha)$. By the minimality for the cardinality of $S$, we see that for any $\alpha, \beta \in B_{S}, s_{\alpha, w_{S}(\alpha)}^{k_{S}(\alpha)}=s_{\beta, w_{S}(\beta)}^{k_{S}(\beta)}$ in $S$ if and only if $\alpha=\beta$. By properly choosing generators in a presentation $(S, \mathcal{P})$ for $G$, 
we can always make an associated simple root system $\left(B_{S}, w_{S}\right)$ to satisfy $k_{S}(\alpha)=1$ for all $\left.\alpha \in B_{S}\right)$

Lemma. Two presentations of $G$ are congruent if and only if their associated simple root systems are equivalent (see 1.7).

Proof. It follows directly from the definitions.

Remark 1.12. (1) In the present paper, we shall deal with Problems A and B (see Introduction) for the groups $G=G_{12} G_{24}, G_{25}$ and $G_{26}$. As a basic work in our method, we calculate the group $\left\langle s_{\alpha}, s_{\beta}\right\rangle$ (or the value $n\left(s_{\alpha}, s_{\beta}\right)$ when $f(\alpha)=f(\beta)=2$ ) for any pair of reflections $s_{\alpha} \neq s_{\beta}$ in $G$ with respect to $\alpha, \beta \in R$, and also calculate all the permutations of $R$ given rise by the action of the reflections $s_{\alpha}, \alpha \in R$ (these results are not included explicitly in the paper). These can be done by computer in general.

(2) Howlett classified all the non-congruent simple reflection sets for the groups $G_{12}$ and $G_{24}$ by a certain detailed analysis on the group-theoretic structure of these groups in a private communication. The method used in the paper is quite different from Howlett's, and could be applied without essential change for the other finite complex reflection groups.

\section{$\S 2$. Simple root systems for the group $G_{12}$ •}

2.1. Let $\epsilon_{1}, \epsilon_{2}$ be an orthonormal basis in a hermitian space $V$ of dimension 2. Let $e_{1}=\epsilon_{1}$ and $e_{2}=\frac{-1+\sqrt{2} i}{2} \epsilon_{1}+\frac{\sqrt{3}-\sqrt{6} i}{6} \epsilon_{2}$. Then $e_{1}, e_{2}$ is also a basis of $V$. Denote by $(a, b)$ a vector $a e_{1}+b e_{2}$ in $V$. Let $R_{12}=\left\{ \pm e_{i} \mid 1 \leqslant i \leqslant 12\right\}$, where

$$
\begin{aligned}
& e_{1}=(1,0), \quad e_{2}=(0,1), \quad e_{3}=(1,1), \quad e_{4}=(1-\sqrt{2} i, 1), \\
& e_{5}=(-\sqrt{2} i, 1), \quad e_{6}=(1,1+\sqrt{2} i), \quad e_{7}=(1, \sqrt{2} i), \quad e_{8}=(\sqrt{2} i,-1+\sqrt{2} i), \\
& e_{9}=(1+\sqrt{2} i, \sqrt{2} i), \quad e_{10}=(1+\sqrt{2} i,-1+\sqrt{2} i), \quad e_{11}=(1-\sqrt{2} i, 2), \quad e_{12}=(2,1+\sqrt{2} i) .
\end{aligned}
$$

Let $s_{i}$ be the reflection in $V$ with respect to $e_{i}$ for $1 \leqslant i \leqslant 12$. Then the action of $s_{i}$ on $e_{j}$, $1 \leqslant i, j \leqslant 3$, are as follows.

\begin{tabular}{|c|c|c|c|}
\hline$e_{i}$ & $s_{1}\left(e_{i}\right)$ & $s_{2}\left(e_{i}\right)$ & $s_{3}\left(e_{i}\right)$ \\
\hline$e_{1}$ & $-e_{1}$ & $e_{6}$ & $e_{8}$ \\
\hline$e_{2}$ & $e_{4}$ & $-e_{2}$ & $-e_{9}$ \\
\hline$e_{3}$ & $e_{5}$ & $e_{7}$ & $-e_{3}$ \\
\hline
\end{tabular}


The group generated by the set $S_{1}=\left\{s_{1}, s_{2}, s_{3}\right\}$ is $G_{12}$ according to the notation by Shephard-Todd (see [5] and [7]). $R_{12}$ is a root system for $G_{12}$ with $B_{1}=\left\{e_{1}, e_{2}, e_{3}\right\}$ a simple root system by [1] or [7].

2.2. By Proposition 1.8, we see that any simple root system $B$ for the group $G_{12}$ contains exactly three vectors. Since $R_{12}$ is transitive under $G_{12}$, we may assume $e_{1} \in B$ up to $G_{12^{-}}$ action. Consider all the possible orders $o\left(s_{1} s_{i}\right)$ of the product $s_{1} s_{i}$ for $1<i \leqslant 12$. Then $o\left(s_{1} s_{11}\right)=2, o\left(s_{1} s_{i}\right)$ is 3 for $i \in\{6,8,10,12\}, 4$ for $i \in\{7,9\}$ and 6 for $i \in\{2,3,4,5\}$. Let $G_{B}$ be the group generated by the reflections with respect to the vectors in $B$. It is easily checked that no $B \subset R_{12}$ with $e_{1}, e_{11} \in B$ and $|B|=3$ satisfies the equation $G_{B} B=R_{12}$. So no simple root system for $G_{12}$ contains a pair of orthogonal roots. We can also show that any $B \in\left\{\left\{e_{1}, e_{6}, e_{10}\right\},\left\{e_{1}, e_{8}, e_{12}\right\}\right\}$ satisfies the condition $G_{B} B \varsubsetneqq R_{12}$. So in a simple root system there must exist some pair of roots $\alpha, \beta$ with $n\left(s_{\alpha}, s_{\beta}\right)>3$ (see 1.9).

2.4. We can show that any of the following $B_{i} \subset R_{12}$ satisfies the equation $G_{B_{i}} B_{i}=R_{12}$ and hence is a simple root system for $G_{12}$ by Proposition 1.8 .

$$
B_{1}=\left\{e_{1}, e_{2}, e_{3}\right\}, \quad B_{2}=\left\{e_{1}, e_{2}, e_{10}\right\}, \quad B_{3}=\left\{e_{1}, e_{2}, e_{7}\right\}, \quad B_{4}=\left\{e_{1}, e_{2}, e_{8}\right\}, \quad B_{5}=\left\{e_{1}, e_{3}, e_{8}\right\}
$$

Recall the notation $\mathbf{n}(B)$ in 1.10. We have $\mathbf{n}\left(B_{1}\right)=(6,6,6), \mathbf{n}\left(B_{2}\right)=(6,3,3), \mathbf{n}\left(B_{3}\right)=$ $(6,3,4), \mathbf{n}\left(B_{4}\right)=(6,4,6)$ and $\mathbf{n}\left(B_{5}\right)=(4,3,3)$. This implies that $B_{i}, 1 \leqslant i \leqslant 5$, are five inequivalent simple root systems for $G_{12}$. By 1.6 and by the knowledge of the values $n\left(s_{\alpha}, s_{\beta}\right)$ for all the non-proportional pairs $\alpha, \beta$ in $R_{12}$, we can further show that there is no more inequivalent simple root system for $G_{12}$ at all.

\section{$\S 3$. Presentations of the group $G_{12}$.}

In this section, we shall find all the non-congruent presentations of the complex reflection group $G_{12}$ by generators and relations according to the results in 2.4 (see Propositions 3.2$3.6)$.

3.1. Suppose that we are given a complex reflection group $G$, the associated root system $R$ of $G$, and all the inequivalent simple root systems $B_{k}, 1 \leqslant k \leqslant r$, for $R$. The strategy for finding all the non-congruent presentations of $G$ is as follows. We start with the presentation of $G$ given by Shephard-Todd which corresponds to a simple root system, say $B_{1}$, for $R$. Let $S_{k}$ be the reflection set associated to the simple root system $B_{k}$. We make use of the relations among the simple root systems $B_{k}, 1 \leqslant k \leqslant r$, to establish some transition 
among the $S_{k}$ 's by Lemma 1.3. Then for each $1<k \leqslant r$, we get a presentation of $G$ with the generator set $S_{k}$ by some related transition. Suppose that we are given a presentation $\left(S_{h}, \mathcal{P}\right)$ of $G$ with the generator set $S_{h}$ and the transition between the sets $S_{h}$ and $S_{k}$. Then the simplest method to produce a presentation of $G$ with the generator set $S_{k}$ is just to substitute the reflections of $S_{h}$ by those of $S_{k}$ in the relations of $\mathcal{P}$ by the related transition. But it may happen that sometimes the resulting relations we get in the new presentation of $G$ are quite complicated and hence are not very useful in practice. So we shall not always use such a method.

3.2. It is well known that the group $G_{12}$ is presented by the generator set $S=\{s, u, t\}$ and the relations (see [1]):

(1) $s^{2}=u^{2}=t^{2}=1$.

(2) suts $=u t s u=t s u t$.

The reflections $s_{1}, s_{2}$ and $s_{3}$ in $V$ satisfy relations (1), (2) with $s_{1}, s_{2}, s_{3}$ in the places of $s, u, t$ respectively. Since $B_{1}=\left\{e_{1}, e_{2}, e_{3}\right\}$ is a simple root system for $G_{12}$, the assignment $\rho(s)=s_{1}, \rho(u)=s_{2}$ and $\rho(t)=s_{3}$ determines a faithful representation $\rho$ of the group $G_{12}$ to $\mathrm{GL}(V)$. This implies part (1) of the following

Proposition 3.3. (1) The group $G_{12}$ can be presented by the generator set $S_{1}=\left\{s_{1}, s_{2}, s_{3}\right\}$ and the relations:

$$
\begin{aligned}
& \text { (a1) } s_{1}^{2}=s_{2}^{2}=s_{3}^{2}=1 . \\
& \text { (a2) } s_{1} s_{2} s_{3} s_{1}=s_{2} s_{3} s_{1} s_{2} . \\
& \text { (a3) } s_{1} s_{2} s_{3} s_{1}=s_{3} s_{1} s_{2} s_{3} .
\end{aligned}
$$

(2) Under the presentation of $G_{12}$ in (1), we have

$$
\begin{aligned}
& \text { (a } s_{4} s_{1} s_{2} s_{1} s_{2} s_{1} s_{2}=s_{2} s_{1} s_{2} s_{1} s_{2} s_{1} . \\
& \text { (a5) } s_{2} s_{3} s_{2} s_{3} s_{2} s_{3}=s_{3} s_{2} s_{3} s_{2} s_{3} s_{2} . \\
& \text { (a6) } s_{1} s_{3} s_{1} s_{3} s_{1} s_{3}=s_{3} s_{1} s_{3} s_{1} s_{3} s_{1} . \\
& \text { (ar) o } o\left(s_{1} s_{2} s_{3}\right)=8 .
\end{aligned}
$$

Proof. We need only show (2). Let $x=s_{1} s_{2} s_{3} s_{1}$ and $y=s_{1} s_{2} s_{3}$. Then $x^{6}=y^{8}$ and $o(x)=o\left(s_{1} s_{2}\right)=o\left(s_{1} s_{3}\right)=o\left(s_{2} s_{3}\right)$ by (a1)-(a3). Thus to show (a4)-(a7), it suffices to show that $x^{6}=1, x^{2} \neq 1$ and $x^{3} \neq 1$, where 1 is the identity transformation in $V$. These can be shown by observing the action of $x^{6}, x^{2}, x^{3}$ on the basis elements $e_{1}, e_{2}$ in $V$. 
3.4. We have, by Lemma $1.3(2)$, that

$$
s_{10}=s_{2} s_{3} s_{2} s_{3} s_{2} \quad \text { and } \quad s_{3}=s_{1} s_{2} s_{10} s_{1} s_{10} s_{2} s_{1}
$$

Proposition. (1) The group $G_{12}$ can be presented by the generator set $S_{2}=\left\{s_{1}, s_{2}, s_{10}\right\}$ and the relations:

(b1) $s_{1}^{2}=s_{2}^{2}=s_{10}^{2}=1$.

(b2) $s_{1} s_{2} s_{1} s_{2} s_{1} s_{2}=s_{2} s_{1} s_{2} s_{1} s_{2} s_{1}$.

(b3) $s_{1} s_{10} s_{1}=s_{10} s_{1} s_{10}$.

(b4) $s_{1} s_{10} s_{2} \cdot s_{1}=s_{2} \cdot s_{1} s_{10} s_{2}$.

(2) Under the presentation of $G_{12}$ in (1), we have

(b5) $s_{2} s_{10} s_{2}=s_{10} s_{2} s_{10}$.

Proof. (b5) follows from (b1), (b3) and (b4). This shows (2) under the assumption of (1). Now we show (1). We must show that relations (a1)-(a3) in Proposition 3.3 are equivalent to (b1)-(b4) here under transition (3.4.1). Clearly, (a1) is equivalent to (b1). So it remains to show that relations (a2)-(a3) are equivalent to (b2)-(b4) under the assumption of (3.4.1) and (a1) (hence (b1)).

(i) First assume (a2)-(a3). Hence (a4)-(a7) hold by Proposition 3.3 (2). Now (b2) is the same as (a4). By (a2)-(a3), we have

$$
s_{3} s_{2} s_{3} s_{1}=s_{1} s_{2} s_{3} s_{2}
$$

(b4) is amount to

$$
s_{1} \cdot s_{2} s_{3} s_{2} s_{3} s_{2} \cdot s_{2} s_{1} s_{2} \cdot s_{2} s_{3} s_{2} s_{3} s_{2} \cdot s_{1} s_{2}=1
$$

By (3.4.2) and (a1)-(a7), LHS of (3.4.3) is equal to

$$
\begin{aligned}
& s_{1} s_{2} s_{3} s_{2} s_{3} s_{1} s_{3} s_{2} s_{3} s_{2} s_{1} s_{2}=s_{1} s_{2} s_{1} s_{2} s_{3} s_{2} s_{3} s_{2} s_{3} s_{2} s_{1} s_{2} \\
= & s_{1} s_{2} s_{1} \cdot s_{3} s_{2} s_{3} s_{2} s_{3} \cdot s_{1} s_{2}=s_{1} s_{2} s_{1} s_{3} s_{2} s_{1} s_{2} s_{3}=s_{1} s_{2} \cdot s_{2} s_{1} s_{3} s_{2} \cdot s_{2} s_{3}=1 .
\end{aligned}
$$

So (3.4.3) follows and hence we get (b4).

For (b3), we need show

$$
s_{1} \cdot s_{2} s_{3} s_{2} s_{3} s_{2} \cdot s_{1} \cdot s_{2} s_{3} s_{2} s_{3} s_{2} \cdot s_{1} \cdot s_{2} s_{3} s_{2} s_{3} s_{2}=1 \text {. }
$$


By (3.4.2) and (a1)-(a7), LHS of (3.4.4) becomes

$$
\begin{aligned}
& s_{1} s_{2} s_{3} \cdot s_{1} s_{3} s_{2} s_{3} \cdot s_{2} s_{3} s_{2} s_{3} s_{2} \cdot s_{3} s_{2} s_{3} s_{1} \cdot s_{3} s_{2} \\
= & s_{1} s_{2} s_{3} s_{1} \cdot s_{2} s_{1} s_{3} s_{2}=s_{1} s_{2} s_{3} s_{1} \cdot s_{1} s_{3} s_{2} s_{1}=1 .
\end{aligned}
$$

This shows (3.4.4) and hence (b3) follows.

(ii) Next assume (b2)-(b4). Hence (b5) holds also. To show (a2)-(a3), we must show the following two equations:

$$
\begin{gathered}
s_{1} s_{2} \cdot s_{1} s_{2} s_{10} s_{1} s_{10} s_{2} s_{1} \cdot s_{1}=s_{2} \cdot s_{1} s_{2} s_{10} s_{1} s_{10} s_{2} s_{1} \cdot s_{1} s_{2} \\
s_{1} s_{2} \cdot s_{1} s_{2} s_{10} s_{1} s_{10} s_{2} s_{1} \cdot s_{1}=s_{1} s_{2} s_{10} s_{1} s_{10} s_{2} s_{1} \cdot s_{1} s_{2} \cdot s_{1} s_{2} s_{10} s_{1} s_{10} s_{2} s_{1} .
\end{gathered}
$$

Now by (b1)-(b5), we have

$$
\begin{aligned}
(3.4 .5) & \Longleftrightarrow s_{1} s_{2} \cdot s_{1} s_{2} s_{10} s_{1} s_{10} s_{2} \cdot s_{10} s_{1} s_{10} s_{2} s_{1} \cdot s_{2}=1 \\
& \Longleftrightarrow s_{1} s_{2} s_{1} s_{2} \cdot s_{1} s_{10} s_{1} s_{2} s_{1} s_{10} s_{1} \cdot s_{2} s_{1} s_{2}=1 \\
& \Longleftrightarrow s_{1} s_{2} s_{1} s_{2} s_{1} s_{2} s_{1} s_{2} \cdot s_{1} s_{2} s_{1} s_{2}=1 .
\end{aligned}
$$

The last equation follows by (b2) and hence we get (3.4.5).

Finally, by (b1)-(b5), we have

$$
\begin{aligned}
(3.4 .6) & \Longleftrightarrow s_{1} s_{2} s_{10} s_{1} s_{10} s_{2} \cdot s_{1} s_{2} s_{10} s_{1} s_{10} s_{2} s_{1} \cdot s_{10} s_{1} s_{10}=1 \\
& \Longleftrightarrow s_{1} s_{2} s_{10} s_{1} \cdot s_{1} s_{2} s_{1} \cdot s_{1} s_{10} s_{2} s_{1} \cdot s_{1} s_{10} s_{1}=1 \\
& \Longleftrightarrow s_{2} s_{10} s_{2} s_{10} s_{2} s_{10}=1 .
\end{aligned}
$$

The last equation follows by (b5) and hence (3.4.6) is shown.

3.5. We have

$$
s_{7}=s_{2} s_{3} s_{2} \quad \text { and } \quad s_{3}=s_{2} s_{7} s_{2} .
$$

Proposition. (1) The group $G_{12}$ can be presented by the generator set $S_{3}=\left\{s_{1}, s_{2}, s_{7}\right\}$ and the relations:

$$
\text { (c1) } s_{1}^{2}=s_{2}^{2}=s_{7}^{2}=1 \text {. }
$$




$$
\begin{aligned}
& \text { (c2) } s_{1} s_{2} s_{7} \cdot s_{1}=s_{2} \cdot s_{1} s_{2} s_{7} . \\
& \text { (c3) } s_{7} s_{2} s_{1} \cdot s_{7}=s_{2} \cdot s_{7} s_{2} s_{1} .
\end{aligned}
$$

(2) Under the presentation of $G_{12}$ in (1), we have

(c4) $s_{1} s_{7} s_{1} s_{7}=s_{7} s_{1} s_{7} s_{1}$.

(c5) $s_{1} s_{2} s_{1} s_{2} s_{1} s_{2}=s_{2} s_{1} s_{2} s_{1} s_{2} s_{1}$.

(c6) $s_{2} s_{7} s_{2} s_{7} s_{2} s_{7}=s_{7} s_{2} s_{7} s_{2} s_{7} s_{2}$.

Proof. (1) We must show that relations (a1)-(a3) in Proposition 3.3 are equivalent to (c1)(c3) here under transition (3.5.1).

By (3.5.1), relation (a1) is equivalent to (c1). Under the assumption of (3.5.1) and (a1) (hence $(\mathrm{c} 1))$, we have

$$
\begin{aligned}
(a 2) & \Longleftrightarrow s_{1} s_{2} \cdot s_{2} s_{7} s_{2} \cdot s_{1}=s_{2} \cdot s_{2} s_{7} s_{2} \cdot s_{1} s_{2} \\
& \Longleftrightarrow s_{1} s_{7} s_{2} s_{1}=s_{7} s_{2} s_{1} s_{2} \Longleftrightarrow(c 2)
\end{aligned}
$$

Then under the assumption of relations (a1)-(a2) (hence (c1)-(c2)), we can show that relation (a3) is equivalent to (c3).

(c5) is the same as (a4). So (c5) follows from (c1)-(c3) by the equivalence between (a1)-(a3) and (c1)-(c3). Then one can easily deduce the equations

$$
s_{1} s_{7} s_{1} s_{7}=s_{1} s_{2} s_{1} s_{2} s_{1} s_{2}=s_{2} s_{7} s_{2} s_{7} s_{2} s_{7} .
$$

from (c1)-(c3). So (c4) and (c6) follow from (c5) and (c1).

3.6. We have

$$
s_{8}=s_{3} s_{1} s_{3}, \quad \text { and } \quad s_{3}=s_{1} s_{2} s_{8} s_{2} s_{1} .
$$

Proposition. (1) The group $G_{12}$ can be presented by the generator set $S_{4}=\left\{s_{1}, s_{2}, s_{8}\right\}$ and the relations:

(d1) $s_{1}^{2}=s_{2}^{2}=s_{8}^{2}=1$.

(d2) $s_{2} s_{1} s_{2} s_{8} \cdot s_{2}=s_{1} \cdot s_{2} s_{1} s_{2} s_{8}$.

(d3) $s_{8} \cdot s_{1} s_{2} s_{8} s_{2}=s_{1} s_{2} s_{8} s_{2} \cdot s_{1}$.

(2) Under the presentation (1) of $G_{12}$, we have

(d4) $s_{1} s_{8} s_{1}=s_{8} s_{1} s_{8}$. 
(d5) $s_{2} s_{8} s_{2} s_{8}=s_{8} s_{2} s_{8} s_{2}$.

(d6) $s_{1} s_{2} s_{1} s_{2} s_{1} s_{2}=s_{2} s_{1} s_{2} s_{1} s_{2} s_{1}$.

Proof. For (1), we must show that relations (a1)-(a3) in Proposition 3.3 are equivalent to (d1)-(d3) here. (a1) is equivalent to (d1) by (3.6.1). Then under relations (3.6.1) and (a1) (hence $(\mathrm{d} 1)$ ), we have

$$
\begin{aligned}
& (a 2) \Longleftrightarrow s_{1} s_{2} s_{1} s_{2} s_{8} s_{2}=s_{2} s_{1} s_{2} s_{8} \Longleftrightarrow(\mathrm{d} 2) \\
& (a 3) \Longleftrightarrow s_{1} s_{2} s_{1} s_{2} s_{8} s_{2}=s_{1} s_{2} s_{8} s_{1} s_{2} s_{8} s_{2} s_{1} \Longleftrightarrow(\mathrm{d} 3) .
\end{aligned}
$$

This shows (1). Now we show (d4)-(d6) under the presentation (1) of $G_{12} \cdot(\mathrm{d} 6)$ is the same as (a4). Rewrite (d2) as

$$
s_{1} s_{2} s_{1} s_{2} s_{1} s_{2}=s_{2} s_{8} s_{2} s_{8}
$$

by (d1), this implies (d5)-(d6) from (d1)-(d3) by the equivalence between (a1)-(a3) and (d1)-(d3). Rewrite (d3) as

$$
s_{1} s_{8} s_{1}=s_{2} s_{8} s_{2} s_{1} s_{2} s_{8} s_{2}
$$

Then to show (d4), it suffices to show that

$$
s_{8} s_{1} s_{8}=s_{2} s_{8} s_{2} s_{1} s_{2} s_{8} s_{2} .
$$

That is to show

$$
s_{1} \cdot s_{2} s_{8} s_{2} s_{8}=s_{2} s_{8} s_{2} s_{8} \cdot s_{1}
$$

which follows by (3.6.2) and (d6). Hence (d4) is shown.

3.7. We have

$$
s_{8}=s_{1} s_{7} s_{2} s_{7} s_{1} \quad \text { and } \quad s_{2}=s_{7} s_{1} s_{8} s_{1} s_{7} .
$$


Proposition. The group $G_{12}$ can be presented by the generator set $S_{5}=\left\{s_{1}, s_{7}, s_{8}\right\}$ and the relations:

$$
\begin{aligned}
& \text { (e1) } s_{1}^{2}=s_{7}^{2}=s_{8}^{2}=1 . \\
& \text { (e2) } s_{1} s_{7} s_{1} s_{7}=s_{7} s_{1} s_{7} s_{1} . \\
& \text { (e3) } s_{1} s_{8} s_{1}=s_{8} s_{1} s_{8} . \\
& \text { (e4) } s_{7} s_{8} s_{7}=s_{8} s_{7} s_{8} . \\
& \text { (e5) } s_{1} s_{7} s_{1} s_{7} \cdot s_{8}=s_{8} \cdot s_{1} s_{7} s_{1} s_{7} .
\end{aligned}
$$

Proof. We must show that relations (c1)-(c3) are equivalent to (e1)-(e5) under transition (3.7.1). Clearly, (c1) is equivalent to (e1). It remains to show that relations (c2)-(c3) are equivalent to (e2)-(e5) under the assumption of (3.7.1) and (c1) (hence (e1)).

(i) First assume (c2)-(c3). Hence (c4)-(c6) hold by Proposition 3.5 (2). (e2) is the same as (c4). (e3) is equivalent to

$$
s_{1} \cdot s_{1} s_{7} s_{2} s_{7} s_{1} \cdot s_{1} \cdot s_{1} s_{7} s_{2} s_{7} s_{1} \cdot s_{1} \cdot s_{1} s_{7} s_{2} s_{7} s_{1}=1
$$

By (c1)-(c4), LHS of (3.7.2) is equal to

$$
\begin{aligned}
& s_{7} s_{2} s_{7} s_{1} \cdot s_{7} s_{2} s_{7} s_{1} \cdot s_{7} s_{2} s_{7} s_{1}=s_{7} s_{2} s_{1} \cdot s_{7} s_{1} s_{7} s_{1} \cdot s_{2} s_{7} s_{1} \cdot s_{7} s_{2} s_{7} s_{1} \\
= & s_{2} s_{7} s_{2} s_{1} \cdot s_{1} \cdot s_{1} s_{2} s_{7} s_{2} s_{1} \cdot s_{7} s_{2} s_{7} s_{1}=s_{2} s_{7} s_{2} s_{1} \cdot s_{7} s_{2} s_{1} \cdot s_{2} s_{7} s_{1} \\
= & s_{7} s_{2} s_{1} \cdot s_{1} s_{2} s_{7}=1 .
\end{aligned}
$$

This implies (e3). (e4) can be shown similarly. Finally, (e5) follows from (c6) by substitution of $s_{2}=s_{7} s_{1} s_{8} s_{1} s_{7}$.

(ii) Next assume (e2)-(e5). By (3.7.1), we get from (e5) that

$$
s_{1} s_{7} s_{1} s_{7} \cdot s_{1} s_{7} s_{2} s_{7} s_{1}=s_{1} s_{7} s_{2} s_{7} s_{1} \cdot s_{1} s_{7} s_{1} s_{7}
$$

and hence

$$
s_{1} s_{7} s_{1} s_{7} \cdot s_{2}=s_{2} \cdot s_{1} s_{7} s_{1} s_{7} .
$$

By (3.7.1) and (3.7.3), we get

$$
\begin{aligned}
(e 3) & \Longrightarrow s_{1} \cdot s_{7} s_{2} s_{7} \cdot s_{1}=s_{7} s_{2} s_{7} \cdot s_{1} \cdot s_{7} s_{2} s_{7} \\
& \Longrightarrow s_{1} s_{7} s_{1} s_{7} \cdot s_{2} \cdot s_{7} s_{1} s_{7}=s_{1} s_{2} \cdot s_{7} s_{1} s_{7} \cdot s_{2} \\
& \Longrightarrow s_{2} \cdot s_{1} s_{7} s_{1} s_{7} \cdot s_{7} s_{1} s_{7}=s_{1} s_{2} \cdot s_{7} s_{1} s_{7} \cdot s_{2} \\
& \Longrightarrow s_{1} \cdot s_{7} s_{2} s_{1}=s_{7} s_{2} s_{1} \cdot s_{2} \\
& \Longrightarrow(c 2)
\end{aligned}
$$


Similarly, (c3) follows from (e4) by (3.7.1) and (3.7.3).

\section{$\S 4$. Simple root systems and presentations for the group $G_{24}$.}

In this section, we shall find all the inequivalent simple root systems for the complex reflection group $G_{24}$ (see 4.2). Then we shall give all the non-congruent presentations of the group $G_{24}$ by generators and relations according to these inequivalent simple root systems (see Propositions 4.3-4.5).

4.1. Let $V$ be a hermitian space of dimension 3 with an orthonormal basis $\epsilon_{1}, \epsilon_{2}, \epsilon_{3}$. Then $e_{1}=\epsilon_{2}, e_{2}=\frac{1}{2}\left(-\epsilon_{1}+\epsilon_{2}+\alpha \epsilon_{3}\right)$ and $e_{3}=\frac{\bar{\alpha}}{2}\left(\epsilon_{2}+\epsilon_{3}\right)$ also form a basis of $V$, where $\bar{\alpha}$ denotes the complex conjugate of $\alpha=\frac{1}{2}(1+i \sqrt{7})$, the latter is a root of the equation $x^{2}-x+2=0$. Denote by $(a, b, c)$ a vector $a e_{1}+b e_{2}+c e_{3}$. Let $R_{24}=\left\{ \pm e_{i} \mid 1 \leqslant i \leqslant 21\right\}$, where

$$
\begin{array}{llll}
e_{1}=(1,0,0) & e_{2}=(0,1,0) & e_{3}=(0,0,1) & e_{4}=(-1,1,0) \\
e_{5}=(-\bar{\alpha}, 0,1) & e_{6}=(0, \alpha, 1) & e_{7}=(1,0,-\alpha) & e_{8}=(0,1, \bar{\alpha}) \\
e_{9}=(-1,1,1) & e_{10}=(-\bar{\alpha}, 1,1) & e_{11}=(-1, \alpha, 1) & e_{12}=(1, \bar{\alpha},-\alpha) \\
e_{13}=(\alpha, 1, \bar{\alpha}) & e_{14}=(-\bar{\alpha}, 1,1+\bar{\alpha}) & e_{15}=(-1, \alpha, 1+\alpha) & e_{16}=(\alpha, \bar{\alpha},-\alpha) \\
e_{17}=(1, \bar{\alpha},-1-\alpha) & e_{18}=(\alpha, 1,1+\bar{\alpha}) & e_{19}=(-\bar{\alpha}, 2,1+\bar{\alpha}) & e_{20}=(-2, \alpha, 1+\alpha) \\
e_{21}=(\alpha, \bar{\alpha}, \bar{\alpha}) . & & &
\end{array}
$$

Denote by $s_{i}$ the reflection in $V$ with respect to the vector $e_{i}, 1 \leqslant i \leqslant 21$. The action of $s_{i}$ on $e_{j}, i, j=1,2,3$, are as follows.

\begin{tabular}{|c|c|c|c|}
\hline$e_{i}$ & $s_{1}\left(e_{i}\right)$ & $s_{2}\left(e_{i}\right)$ & $s_{3}\left(e_{i}\right)$ \\
\hline$e_{1}$ & $-e_{1}$ & $-e_{4}$ & $e_{7}$ \\
\hline$e_{2}$ & $e_{4}$ & $-e_{2}$ & $e_{8}$ \\
\hline$e_{3}$ & $e_{5}$ & $e_{6}$ & $-e_{3}$ \\
\hline
\end{tabular}

The group generated by $s_{1}, s_{2}, s_{3}$ is $G_{24}$. $R_{24}$ is the root system for $G_{24}$ with $B_{1}=$ $\left\{e_{1}, e_{2}, e_{3}\right\}$ a simple root system (see [7]).

4.2. We want to find all the inequivalent simple root systems $B_{k}, k \geqslant 1$, for $G_{24}$. By Proposition 1.8, we have $\left|B_{k}\right|=3$. Since $R_{24}$ is transitive under $G_{24}$, we may assume $e_{1} \in B_{k}$ up to $G_{24}$-action. The number $n\left(s_{1}, s_{i}\right)$ (see 1.9) is 2 for $i \in\{7,17,19,21\}, 3$ for $i \in\{2,4,6,11,14,15,18,20\}$ and 4 for $i \in\{3,5,8,9,10,12,13,16\}$.

Any finite reflection group generated by $s_{1}, s_{i}, s_{j}$ with $i \in\{7,17,19,21\}$ is a homomorphic image of the Coxeter group of type $A_{3}, B_{3}$ or $\widetilde{C}_{2}$, hence it has order $\leqslant 48$ and is not 
isomorphic to $G_{24}\left(G_{24}\right.$ has order 336). So a simple root system $B_{k}$ for $G_{24}$ contains no pair of orthogonal roots. By 1.6, Proposition 1.8 and the knowledge of the values $n\left(s_{i}, s_{j}\right)$, $1 \leqslant i, j \leqslant 21$, we see that the following are all the inequivalent simple root systems for $R_{24}$ :

$$
B_{1}=\left\{e_{1}, e_{2}, e_{3}\right\}, \quad B_{2}=\left\{e_{1}, e_{3}, e_{4}\right\} \quad \text { and } \quad B_{3}=\left\{e_{1}, e_{3}, e_{8}\right\}
$$

We have $\mathbf{n}\left(B_{1}\right)=(3,4,4), \mathbf{n}\left(B_{2}\right)=(4,3,3)$ and $\mathbf{n}\left(B_{3}\right)=(4,4,4)$ (see 1.10).

4.3. Next we shall find all the non-congruent presentations of $G_{24}$ by generators and relations.

Proposition. The group $G_{24}$ can be presented by the generator set $S_{1}=\left\{s_{1}, s_{2}, s_{3}\right\}$ and the relations:

$$
\begin{aligned}
& \text { (a1) } s_{1}^{2}=s_{2}^{2}=s_{3}^{2}=1 . \\
& \text { (a2) } s_{1} s_{3} s_{1} s_{3}=s_{3} s_{1} s_{3} s_{1} . \\
& \text { (a3) } s_{1} s_{2} s_{1}=s_{2} s_{1} s_{2} . \\
& \text { (a4) } s_{2} s_{3} s_{2} s_{3}=s_{3} s_{2} s_{3} s_{2} . \\
& \text { (a5) } s_{3} \cdot s_{2} s_{1} s_{2} s_{3} s_{2}=s_{2} s_{1} s_{2} s_{3} s_{2} \cdot s_{1} .
\end{aligned}
$$

Proof. This follows by [1, Table 4] and by the argument as for Proposition 3.3 (1).

4.4. We have

$$
s_{4}=s_{1} s_{2} s_{1} \quad \text { and } \quad s_{2}=s_{1} s_{4} s_{1}
$$

Proposition. The group $G_{24}$ can be presented by the generator set $S_{2}=\left\{s_{1}, s_{3}, s_{4}\right\}$ and the relations:

$$
\begin{aligned}
& \text { (b1) } s_{1}^{2}=s_{3}^{2}=s_{4}^{2}=1 . \\
& \text { (b2) } s_{1} s_{3} s_{1} s_{3}=s_{3} s_{1} s_{3} s_{1} . \\
& \text { (b3) } s_{1} s_{4} s_{1}=s_{4} s_{1} s_{4} . \\
& \text { (b4) } s_{3} s_{4} s_{3}=s_{4} s_{3} s_{4} . \\
& \text { (b5) } s_{1} s_{3} s_{1} \cdot s_{4} s_{1} s_{3} s_{1} s_{4}=s_{4} s_{1} s_{3} s_{1} s_{4} \cdot s_{1} s_{3} s_{1} .
\end{aligned}
$$

Proof. Under transition (4.4.1), relation (a1) is equivalent to (b1). Also, (a2) is the same as (b2). Then under relations (4.4.1) and (a1) (hence (b1)), relation (a3) (resp. (a4)) is equivalent to (b3) (resp. (b5)). Finally, under relations (4.4.1), (a1), (a3), (b1) and (b3), relation (a5) is equivalent to (b4). So our result follows by Proposition 4.3. 
4.5. We have

$$
s_{8}=s_{3} s_{2} s_{3} \quad \text { and } \quad s_{2}=s_{3} s_{8} s_{3} .
$$

Proposition. (1) The group $G_{24}$ can be presented by the generator set $S_{3}=\left\{s_{1}, s_{3}, s_{8}\right\}$ and the relations:

$$
\begin{aligned}
& \text { (c1) } s_{1}^{2}=s_{3}^{2}=s_{8}^{2}=1 . \\
& \text { (c2) } s_{1} s_{3} s_{1} s_{3}=s_{3} s_{1} s_{3} s_{1} . \\
& \text { (c3) } s_{3} s_{8} s_{3} s_{8}=s_{8} s_{3} s_{8} s_{3} . \\
& \text { (c4) } s_{3} s_{1} s_{3} s_{8} s_{3} \cdot s_{1}=s_{8} \cdot s_{3} s_{1} s_{3} s_{8} s_{3} . \\
& \text { (c5) } s_{8} s_{1} s_{8} s_{3} s_{8} \cdot s_{1}=s_{3} \cdot s_{8} s_{1} s_{8} s_{3} s_{8} .
\end{aligned}
$$

(2) Under the presentation of $G_{24}$ in (1), we have

$$
\text { (c6) } s_{1} s_{8} s_{1} s_{8}=s_{8} s_{1} s_{8} s_{1} \text {. }
$$

Proof. Under transition (4.5.1), relation (a1) is equivalent to (c1). Also, (a2) is the same as (c2). Then under relations (4.5.1) and (a1) (hence (c1)), relation (a4) (resp. (a3)) is equivalent to (c3) (resp. (c4)). Then under relations (a1) and (a4) (hence (c1) and (c3)), relation (a5) is equivalent to (c5). This implies (1). Now we show (2). Right-multiplying both sides of (c4) by the inverse of the corresponding sides of (c5) and then applying (c3), we get

$$
s_{8} s_{1} \cdot s_{3} s_{8} \cdot s_{1} s_{3}=s_{3} s_{8} s_{1} \cdot s_{3} s_{8} \cdot s_{1} s_{3} s_{8}
$$

and hence

$$
s_{3} s_{8} s_{1} s_{3} s_{8} \cdot s_{1} s_{3}=s_{8} s_{1} \cdot s_{3} s_{8} s_{1} s_{3} s_{8}
$$

So for any $k \in \mathbb{N}$, we have

$$
s_{3} s_{8} s_{1} s_{3} s_{8} \cdot\left(s_{1} s_{3}\right)^{k}=\left(s_{8} s_{1}\right)^{k} \cdot s_{3} s_{8} s_{1} s_{3} s_{8}
$$

This implies that $\left(s_{1} s_{3}\right)^{k}=1$ if and only if $\left(s_{8} s_{1}\right)^{k}=1$. So (c6) follows by (c2).

\section{$\S 5$. Simple root systems and presentations for the group $G_{25}$.}

In this section, we deal with the complex reflection group $G_{25}$. Comparing with the groups $G_{12}, G_{24}$, a notable difference of $G_{25}$ is that all of its reflections have order 3, rather than 2. There are two inequivalent simple root systems and two non-congruent presentations 
for $G_{25}$ (see 5.2 and Propositions 5.3, 5.4). The presentation given in Corollary 5.4 is congruent to that in Proposition 5.4.

5.1. Let $V, \epsilon_{i}$ be as in 4.1. Let $e_{1}=\bar{\omega} \epsilon_{3}, e_{2}=3^{-1 / 2} i\left(\epsilon_{1}+\epsilon_{2}+\epsilon_{3}\right)$ and $e_{3}=\bar{\omega} \epsilon_{2}$, where $\omega$ is a primitive cubic root of unity and $\bar{\omega}$ its complex conjugate. Then $B_{1}=\left\{e_{1}, e_{2}, e_{3}\right\}$ forms a basis of $V$. Denote by $(a, b, c)$ a vector $a e_{1}+b e_{2}+c e_{3}$. Let $R_{25}=\left\{(-\omega)^{k} e_{i} \mid 1 \leqslant\right.$ $i \leqslant 12,0 \leqslant k<6\}$, where

$$
\begin{array}{llll}
e_{1}=(1,0,0), & e_{2}=(0,1,0), & e_{3}=(0,0,1), & e_{4}=(-\bar{\omega}, 1,0), \\
e_{5}=(1,1,0), & e_{6}=(0,1,1), & e_{7}=(0,1,-\bar{\omega}), & e_{8}=(-\bar{\omega}, 1,1), \\
e_{9}=(1,1,1), & e_{10}=(1,1,-\bar{\omega}), & e_{11}=(-\bar{\omega}, 1,-\bar{\omega}), & e_{12}=(-\bar{\omega}, 1-\bar{\omega},-\bar{\omega}) .
\end{array}
$$

Let $s_{i}$ be the reflection in $V$ with respect to $e_{i}, 1 \leqslant i \leqslant 12$. Then the group generated by $S_{1}=\left\{s_{1}, s_{2}, s_{3}\right\}$ is the complex reflection group $G_{25}$ (in the notation of [5]). The set $R_{25}$ forms a root system of $G_{25}$ with $B_{1}$ a simple root system (see [7]). The action of $S_{1}$ on $B_{1}$ is as follows.

\begin{tabular}{|c|c|c|c|}
\hline$e_{i}$ & $s_{1}\left(e_{i}\right)$ & $s_{2}\left(e_{i}\right)$ & $s_{3}\left(e_{i}\right)$ \\
\hline$e_{1}$ & $\omega e_{1}$ & $-\omega e_{4}$ & $e_{1}$ \\
\hline$e_{2}$ & $e_{5}$ & $\omega e_{2}$ & $e_{6}$ \\
\hline$e_{3}$ & $e_{3}$ & $-\omega e_{7}$ & $\omega e_{3}$ \\
\hline
\end{tabular}

5.2. We want to find all the inequivalent simple root systems $B_{k}, k \geqslant 1$, for $G_{25}$. By Proposition 1.8, we have $\left|B_{k}\right|=3$. Since $R_{25}$ is transitive under $G_{25}$, we may assume $e_{1} \in B_{k}$ up to $G_{25}$-action. Let $H_{i j}$ be the subgroup of $G_{25}$ generated by $s_{i}, s_{j}$ for $1 \leqslant i \neq j \leqslant 12$. Then by the action on $R_{25}$ of the subgroups $H_{1 i}$ for any $1<i \leqslant 12$, we see that $H_{1 i}$ is isomorphic to the elementary 3 -group $\mathbb{Z}_{3}^{2}$ for $i=3,12$, and is isomorphic to the group $G_{4}$ (in the notation of [5]) for all the other $i$. From this fact, it is easily seen that all the inequivalent simple root systems for $R_{25}$ are as follows.

$B_{1}=\left\{e_{1}, e_{2}, e_{3}\right\}, B_{2}=\left\{e_{1}, e_{2}, e_{6}\right\}$.

We have $\mathbf{n}\left(B_{1}\right)=\left(G_{4}, G_{4}, \mathbb{Z}_{3}^{2}\right)$ and $\mathbf{n}\left(B_{2}\right)=\left(G_{4}, G_{4}, G_{4}\right)$ (see 1.10).

5.3. Next we shall find all the non-congruent presentations of $G_{25}$ by generators and relations according to the result in 5.2.

Proposition. The group $G_{25}$ can be presented by the generator set $S_{1}=\left\{s_{1}, s_{2}, s_{3}\right\}$ and the relations: 


$$
\begin{aligned}
& \text { (a1) } s_{1}^{3}=s_{2}^{3}=s_{3}^{3}=1 \\
& \text { (a2) } s_{1} s_{2} s_{1}=s_{2} s_{1} s_{2} ; \\
& \text { (a3) } s_{2} s_{3} s_{2}=s_{3} s_{2} s_{3} \\
& \text { (a4) } s_{1} s_{3}=s_{3} s_{1} .
\end{aligned}
$$

Proof. This follows by [1, Table 1].

5.4. We have

$$
s_{6}=s_{2}^{-1} s_{3} s_{2} \quad \text { and } \quad s_{3}=s_{2} s_{6} s_{2}^{-1} .
$$

Proposition. (1) The group $G_{25}$ can be presented by the generator set $S_{2}=\left\{s_{1}, s_{2}, s_{6}\right\}$ and the relations:

(b1) $s_{1}^{3}=s_{2}^{3}=s_{6}^{3}=1$;

(b2) $s_{1} s_{2} s_{1}=s_{2} s_{1} s_{2}$;

(b3) $s_{2} s_{6} s_{2}=s_{6} s_{2} s_{6}$;

(b4) $s_{6} s_{1} s_{2} s_{6}=s_{2} s_{6} s_{1} s_{2}$.

(2) Under the presentation of $G_{25}$ in (1), we have

(b5) $s_{1} s_{6} s_{1}=s_{6} s_{1} s_{6}$.

Proof. (a2) is the same as (b2). By transition (5.4.1), relation (a1) (resp. (a3)) is equivalent to (b1) (resp. (b3)). Then under the assumption of relation (a3) (hence (b3)), relation (a4) is equivalent to (b4). So (1) follows by Proposition 5.3. Now we show (2). Left-multiplying $s_{6}^{-1}$ and right-multiplying $s_{1}$ on both sides of (b4) and then applying (b2)-(b3), we get

$$
s_{1} s_{2} s_{6} s_{1}=s_{6}^{-1} s_{2} s_{6} s_{1} s_{2} s_{1}=s_{2} s_{6} s_{2}^{-1} s_{1} s_{2} s_{1}=s_{2} s_{6} s_{1} s_{2} .
$$

This, together with (b4), implies that

$$
s_{1} s_{2} s_{6} s_{1}=s_{6} s_{1} s_{2} s_{6} .
$$

Left-multiplying $s_{1}^{-1} s_{2}^{-1}$ on both sides of (5.4.2) and then applying (b2), we get

$$
s_{2} s_{1}^{-1} s_{6} s_{1}=s_{1}^{-1} s_{6} s_{1} s_{2} .
$$

Then left-multiplying $s_{1}^{-1}$ and right-multiplying $s_{6}^{-1}$ on both sides of (5.4.3), we get

$$
s_{2} s_{6} s_{1} s_{6}^{-1}=s_{1}^{-1} s_{6} s_{1} s_{2} .
$$

Therefore (b5) follows by (5.4.4) and (5.4.5).

With (5.4.3) instead of (b3) in the proposition, we get another congruent presentation of $G_{25}$. 
Corollary. The group $G_{25}$ can be presented by the generator set $S_{2}=\left\{s_{1}, s_{2}, s_{6}\right\}$ and the relations:

$$
\begin{aligned}
& \text { (b1) } s_{1}^{3}=s_{2}^{3}=s_{6}^{3}=1 ; \\
& \text { (b2) } s_{1} s_{2} s_{1}=s_{2} s_{1} s_{2} ; \\
& \text { (b3') } s_{1} s_{2} s_{6} s_{1}=s_{2} s_{6} s_{1} s_{2} ; \\
& \text { (b4) } s_{6} s_{1} s_{2} s_{6}=s_{2} s_{6} s_{1} s_{2} .
\end{aligned}
$$

The proof is left to the readers. Clearly, the presentation in the corollary is congruent to that in the proposition. With (b2) replaced by either (b3) or (b5) in the corollary, we get two more congruent presentations of $G_{25}$. One might notice that relations (b3'), (b4) are similar to (a2), (a3) in Proposition 3.3 for the group $G_{12}$ with $s_{3}$ instead of $s_{6}$. But it should be indicated that the orders of the generators for $G_{25}$ are different from those for $G_{12}$.

\section{$\S$ 6. Simple root systems and presentations for the group $G_{26}$.}

In this section, we do the same for the complex reflection group $G_{26}$. Unlike the previous three groups, the generators of $G_{26}$ have two different orders, and the root system of $G_{26}$ consists of two $G_{26}$-orbits. There are two inequivalent simple root systems and two noncongruent presentations for $G_{26}$ (see 6.2 and Propositions 6.3, 6.4).

6.1. Let $V, \epsilon_{i}$ be as in 4.1. The vectors $e_{1}=2^{-1 / 2}\left(\epsilon_{2}-\epsilon_{3}\right), e_{2}=\epsilon_{3}$ and $e_{3}=3^{-1 / 2} i\left(\epsilon_{1}+\right.$ $\left.\epsilon_{2}+\epsilon_{3}\right)$ form a basis in $V$. Denote by $(a, b, c)$ a vector $a e_{1}+b e_{2}+c e_{3}$. Let $R_{26}=\left\{(-\omega)^{j} e_{i} \mid\right.$ $1 \leqslant i \leqslant 21, j \in J\}$ be with

$$
\begin{aligned}
& e_{1}=(1,0,0), \quad e_{2}=(0,1,0), \quad e_{3}=(0,0,1), \quad e_{4}=(1, y, 0), \\
& e_{5}=(\sqrt{2}, 1,0), \quad e_{6}=(0,1,-\bar{\omega}), \quad e_{7}=(0, \bar{\omega}, 1), \quad e_{8}=(-\omega, y, 0) \text {, } \\
& e_{9}=(1, y,-\bar{\omega} y), \quad e_{10}=(\sqrt{2}, 1,-\bar{\omega}), \quad e_{11}=(\sqrt{2}, 1, \omega), \quad e_{12}=(-\omega, y,-\bar{\omega} y), \\
& e_{13}=(1, y, \omega y), \quad e_{14}=(\sqrt{2}, 1-\omega,-\bar{\omega}), \quad e_{15}=(\sqrt{2}, 2, \omega), \quad e_{16}=(-\omega, y, \omega y), \\
& e_{17}=(1,3 / \sqrt{2}, \omega y), \quad e_{18}=(-\sqrt{2} \omega, 1-\omega,-\bar{\omega}), \quad e_{19}=(\sqrt{2}, 2,-\bar{\omega}), \quad e_{20}=(2,3 / \sqrt{2}, \omega y) \text {, } \\
& e_{21}=(\sqrt{2}, 2, \sqrt{3} i)
\end{aligned}
$$

where $J=\{0,1,2,3,4,5\}$ and $y=\frac{1}{\sqrt{2}}(1-\omega)$. Let $s_{i}$ be the reflection in $V$ with respect to the vector $e_{i}$. Then the action of $s_{i}$ on $e_{j}, i, j=1,2,3$, are as follows. 


\begin{tabular}{|c|c|c|c|}
\hline$e_{i}$ & $s_{1}\left(e_{i}\right)$ & $s_{2}\left(e_{i}\right)$ & $s_{3}\left(e_{i}\right)$ \\
\hline$e_{1}$ & $-e_{1}$ & $e_{4}$ & $e_{1}$ \\
\hline$e_{2}$ & $e_{5}$ & $\omega e_{2}$ & $e_{6}$ \\
\hline$e_{3}$ & $e_{3}$ & $e_{7}$ & $\omega e_{3}$ \\
\hline
\end{tabular}

The group generated by $s_{1}, s_{2}, s_{3}$ is $G_{26}$. $R_{26}$ is the root system for $G_{26}$ with $B_{1}=$ $\left\{e_{1}, e_{2}, e_{3}\right\}$ a simple root system (see [7]).

6.2. Now we consider all the inequivalent simple root systems $B_{k}, k \geqslant 1$. By Proposition 1.8, we have $\left|B_{k}\right|=3$. The set $R_{26}$ consists of two $G_{26}$-orbits: $R_{1}=\left\{\omega^{j} e_{i} \mid i \in I, j \in\right.$ $J\}$ and $R_{2}=\left\{\omega^{j} e_{k} \mid j \in J, k \in K\right\}$, where $I=\{1,4,8,9,12,13,16,17,20\}$ and $K=$ $\{2,3,5,6,7,10,11,14,15,18,19,21\}$. By Lemma 1.3 and condition $G_{26} B_{k}=R_{26}$, we have $B_{k} \cap R_{i} \neq \emptyset$ for $i=1,2$. We may assume $e_{1} \in B_{k}$ up to $G_{26}$-action. By the action of the $s_{i}$ 's on $R_{26}$, we see that each $B_{k}$ contains one root in $R_{1}$ and two roots in $R_{2}$. All the inequivalent simple root systems for $R_{26}$ are as follows.

$B_{1}=\left\{e_{1}, e_{2}, e_{3}\right\}, B_{2}=\left\{e_{1}, e_{2}, e_{6}\right\}$.

We have $\mathbf{n}\left(B_{1}\right)=\left(G(3,1,2), G_{4}, \mathbb{Z}_{2} \times \mathbb{Z}_{3}\right)$ and $\mathbf{n}\left(B_{2}\right)=\left(G(3,1,2), G(3,1,2), G_{4}\right)$ (see 1.10 for $\mathbf{n}(B)$ and $[5]$ for $G(3,1,2))$.

6.3. Next we consider all the non-congruent presentations of $G_{26}$ by generators and relations according to the result in 6.2 .

Proposition. The group $G_{26}$ can be presented by the generator set $S_{1}=\left\{s_{1}, s_{2}, s_{3}\right\}$ and the relations:

$$
\begin{aligned}
& \text { (a1) } s_{1}^{2}=s_{2}^{3}=s_{3}^{3}=1 ; \\
& \text { (a2) } s_{1} s_{2} s_{1} s_{2}=s_{2} s_{1} s_{2} s_{1} \text {; } \\
& \text { (a3) } s_{2} s_{3} s_{2}=s_{3} s_{2} s_{3} ; \\
& \text { (a4) } s_{1} s_{3}=s_{3} s_{1} .
\end{aligned}
$$

Proof. This follows by [1, Table 1].

6.4. We have

$$
s_{6}=s_{2}^{-1} s_{3} s_{2} \quad \text { and } \quad s_{3}=s_{2} s_{6} s_{2}^{-1}
$$

Proposition. (1) The group $G_{26}$ can be presented by the generator set $S_{2}=\left\{s_{1}, s_{2}, s_{6}\right\}$ and the relations:

$$
\text { (b1) } s_{1}^{2}=s_{2}^{3}=s_{6}^{3}=1 \text {; }
$$


(b2) $s_{1} s_{2} s_{1} s_{2}=s_{2} s_{1} s_{2} s_{1}$;

(b3) $s_{2} s_{6} s_{2}=s_{6} s_{2} s_{6}$;

(b4) $s_{2} s_{6} s_{1} s_{2}=s_{6} s_{1} s_{2} s_{6}$.

(2) Under the presentation of $G_{26}$ in (1), we have

(b5) $s_{1} s_{6} s_{1} s_{6}=s_{6} s_{1} s_{6} s_{1}$.

Proof. (1) Relations (a2) and (b2) are the same. By transition (6.4.1), relation (a1) (resp., (a3)) is equivalent to (b1) (resp., (b3)). Then under the assumption of relations (6.4.1) and (b3) (hence (a3)), we have

$$
(\mathrm{a} 4) \Longleftrightarrow s_{1} \cdot s_{2} s_{6} s_{2}^{-1}=s_{2} s_{6} s_{2}^{-1} \cdot s_{1} \Longleftrightarrow s_{1} \cdot s_{2} s_{6} s_{2}^{-1}=s_{6}^{-1} s_{2} s_{6} \cdot s_{1} \Longleftrightarrow(\mathrm{b} 4)
$$

This implies (1). Now we show (2). Rewrite (b4) as

$$
s_{2}^{-1} \cdot s_{6} s_{1} \cdot s_{2}=s_{6} \cdot s_{1} s_{2} \cdot s_{6}^{-1}
$$

Squaring both sides and then applying (b2), we get

$$
s_{2}^{-1} \cdot s_{6} s_{1} s_{6} s_{1} \cdot s_{2}=s_{6} \cdot s_{2} s_{1} s_{2} s_{1} \cdot s_{6}^{-1}
$$

Left-multiplying $s_{2}^{-1} s_{6}^{-1} s_{2}$ and right-multiplying $s_{6} s_{2} s_{6}^{-1}$ on both sides of (6.4.3) and then applying (b3), we get

$$
s_{2}^{-1} \cdot s_{1} s_{6} s_{1} s_{6} \cdot s_{2}=s_{6} \cdot s_{1} s_{2} s_{1} s_{2} \cdot s_{6}^{-1}
$$

So (b5) follows by (b2), (6.4.3) and (6.4.4).

\section{$\S 7$. Essential presentations.}

7.1. According to the definition in 1.9 , if $(S, \mathcal{P})$ is a presentation of a reflection group $G$, and if $\mathcal{P}^{\prime}$ is a set of relations on $S$, including $\mathcal{P}$ as a subset, then $\left(S, \mathcal{P}^{\prime}\right)$ is also a presentation of $G$.

A presentation $(S, \mathcal{P})$ of $G$ is essential if $\left(S, \mathcal{P}_{0}\right)$ is not a presentation of $G$ for any proper subset $\mathcal{P}_{0}$ of $\mathcal{P}$.

Checking the essentiality for a presentation of a group is usually a sublte task.

In a presentation $(S, \mathcal{P})$ of $G$, a relation $P \in \mathcal{P}$ is redundant if $P$ is a consequence of the relations in $\mathcal{P} \backslash\{P\}$. Thus a presentation $(S, \mathcal{P})$ of $G$ is essential if and only if $\mathcal{P}$ contains no redundant relation. 
Let $(S, \mathcal{P})$ be one of the presentations we get so far for the groups $G=G_{12}, G_{24}, G_{25}, G_{26}$. We define $\mathcal{P}^{\text {re }}$, which is obtained from $\mathcal{P}$ in the following way: Let $B$ be a simple root system for $G$ such that $S=\left\{s_{\alpha} \mid \alpha \in B\right\}$. For any $\alpha \in B$, if $G \alpha \cap B$ contains $k>1$ elements, then we remove any $k-1$ of the relations $s_{\beta}^{o\left(s_{\beta}\right)}=1, \beta \in G \alpha \cap B$, from $\mathcal{P}$.

Example 7.2. (1) Let $(S, \mathcal{P})$ be the presentation of $G_{12}$ given in Proposition 3.3. Then $\mathcal{P}^{\text {re }}$ is obtained from $\mathcal{P}$ by removing relations $s_{2}^{2}=1$ and $s_{3}^{2}=1$.

(2) Let $(S, \mathcal{P})$ be the presentation of $G_{26}$ in Proposition 6.3. Then $\mathcal{P}^{\text {re }}$ is obtained from $\mathcal{P}$ by removing relation $s_{3}^{3}=1$.

The main result of the present section is as follows.

Theorem 7.3. For any presentation $(S, \mathcal{P})$ we get so far for the group $G=G_{12}, G_{24}, G_{25}$ or $G_{26}$, the above defined $\left(S, \mathcal{P}^{\mathrm{re}}\right)$ is again a presentation of $G$. Moreover, $\left(S, \mathcal{P}^{\text {re }}\right)$ is essential.

We shall show the theorem in the remaining part of the section. The following is a simple fact in the group theory.

Lemma 7.4. In a group $G$, if $x, y, z \in G$ satisfy $x y=y z$, then $o(x)=o(z)$.

Then the conclusion that $\left(S, \mathcal{P}^{\text {re }}\right)$ is a presentation of the group $G$ follows by Lemma 7.4. In Example $7.2(1)$, relations $s_{2}^{2}=s_{3}^{2}=1$ are the consequence of relations $s_{1}^{2}=1$, $s_{1} \cdot s_{2} s_{3} s_{1}=s_{2} s_{3} s_{1} \cdot s_{2}$ and $s_{3} \cdot s_{1} s_{2} s_{3}=s_{1} s_{2} s_{3} \cdot s_{1}$ in $\mathcal{P}^{\text {re }}$ (see Proposition 3.3, (a1)-(a3)). In Example $7.2(2)$, relation $s_{3}^{3}=1$ is a consequence of relations $s_{2}^{3}=1$ and $s_{2} \cdot s_{3} s_{2}=s_{3} s_{2} \cdot s_{3}$ in $\mathcal{P}^{\text {re }}$ (see Proposition 6.3, (a1)-(a4)). So in each of these two examples, $\left(S, \mathcal{P}^{\text {re }}\right.$ ) is a presentation of the respective group. All the other cases could be checked similarly. Thus it makes sense to call $\left(S, \mathcal{P}^{\text {re }}\right)$ the reduced form for the presentation $(S, \mathcal{P})$.

Let $\left(\mathrm{x} 1^{\mathrm{re}}\right)$ be the set of relation(s) in the presentation $\left(S, \mathcal{P}^{\mathrm{re}}\right)$ obtained from $(\mathrm{x} 1)$ in the presentation $(S, \mathcal{P})$ by removing certain relation $(\mathrm{s})$ for $\mathrm{x}=\mathrm{a}, \mathrm{b}, \mathrm{c}, \ldots$. For example, when $(S, \mathcal{P})$ is the presentation of the group $G_{12}$ (resp., $G_{26}$ ) in Proposition 3.3 (resp., Proposition $6.3),\left(\mathrm{a} 1^{\mathrm{re}}\right)$ in $\left(S, \mathcal{P}^{\mathrm{re}}\right)$ contains a single relation $s_{1}^{2}=1$ (resp., two relations $s_{1}^{2}=1$ and $\left.s_{2}^{3}=1\right)$.

The following fact is useful in checking the essentiality for a presentation of a complex reflection group.

Lemma 7.5. Let $G$ be a complex reflection group with $(S, \mathcal{P})$ its presentation.

(1) The relation set $\mathcal{P}$ contains at least one relation which either involves more than two generators, or has the form $s^{m}=1$ with $m>2$ for some $s \in S$. 
(2) The relation(s) contained in $\left(x 1^{\mathrm{re}}\right), x=a, b, \ldots$, is (are) not redundant in the reduced form of $(S, \mathcal{P})$ when $(S, \mathcal{P})$ is one of those we get in the previous sections.

(3) Let $\left(S, \mathcal{P}^{\prime}\right)$ be another presentation of $G$ with $\mathcal{P}^{\prime} \subseteq \mathcal{P}$. For any $P \in \mathcal{P}^{\prime}$, if $P$ is redundant in $\mathcal{P}^{\prime}$, then $P$ is redundant in $\mathcal{P}$.

Proof. (1) is true since a complex reflection group is not a Coxeter group. Then (2) follows by the following fact: substituting $s_{i}=c$ for all $1 \leqslant i \leqslant 3$ and any fixed $c \in \mathbb{C} \backslash\{0\}$, all the relations in $\mathcal{P} \backslash\{P \mid P \in(x 1)\}$ remain valid. Finally, as a consequence of relations in $\mathcal{P}^{\prime} \backslash\{P\}, P$ is clearly a consequence of relations in $\mathcal{P} \backslash\{P\}$. So we get (3).

Thus it remains to show the essentiality of the reduced form $\left(S, \mathcal{P}^{\text {re }}\right)$ for any presentation $(S, \mathcal{P})$ we get so far for the group $G=G_{12}, G_{24}, G_{25}$ or $G_{26}$. The following result will be used for this purpose.

Lemma 7.6. Suppose that a reflection group $G$ has two presentations $(S, \mathcal{P})$ and $\left(S^{\prime}, \mathcal{P}^{\prime}\right)$, where $\mathcal{P}=\left\{P_{i} \mid i \in I\right\}$ and $\mathcal{P}^{\prime}=\left\{P_{i}^{\prime} \mid i \in I^{\prime}\right\}$ are relation sets with $\left(I, \leqslant_{I}\right),\left(I^{\prime}, \leqslant_{I^{\prime}}\right)$ finite posets (the word "poset "means "partially ordered set"). Suppose that there exists an order-preserving surjection $\phi:\left(I, \leqslant_{I}\right) \longrightarrow\left(I^{\prime}, \leqslant_{I^{\prime}}\right)$ such that for any $k \in I$, relation set $\left\{P_{i} \mid i \leqslant_{I} k\right\}$ is equivalent to $\left\{P_{i^{\prime}}^{\prime} \mid i^{\prime} \leqslant_{I^{\prime}} \phi(k)\right\}$. Suppose that $m, \phi(m)$ are maximal elements in $I, I^{\prime}$, respectively with $\phi^{-1}(\phi(m))=\{m\}$. Then relation $P_{m}$ is not redundant in $\mathcal{P}$ if and only if $P_{\phi(m)}^{\prime}$ is not redundant in $\mathcal{P}^{\prime}$.

Proof. First show the implication " $\Longrightarrow "$. We argue by contrary. Suppose that $P_{\phi(m)}^{\prime}$ is a consequence of all the other relations in $\mathcal{P}^{\prime}$. By our assumption, we see that relation $P_{m}$ is a consequence of the relations in $\left\{P_{i}^{\prime} \mid i \leqslant I^{\prime} \phi(m)\right\}$ and hence a consequence of the relations in $\mathcal{P}^{\prime} \backslash\left\{P_{\phi(m)}^{\prime}\right\}$. Since relation set $\mathcal{P} \backslash\left\{P_{m}\right\}$ is equivalent to relation set $\mathcal{P}^{\prime} \backslash\left\{P_{\phi(m)}^{\prime}\right\}$ by our condition, this implies that $P_{m}$ is a consequence of the relations in $\mathcal{P} \backslash\left\{P_{m}\right\}$, a contradiction.

The implication " $\Longleftarrow "$ can be shown similarly by interchanging the roles of $m, I, \mathcal{P}$ with $\phi(m), I^{\prime}, \mathcal{P}^{\prime}$ respectively.

7.7. By Lemma $7.5(2)$, we see that relation $\left(x 1^{\mathrm{re}}\right), \mathrm{x}=\mathrm{a}, \mathrm{b}, \ldots$, is not redundant in the respective reduced presentation for the group $G=G_{12}, G_{24}, G_{25}$ or $G_{26}$. In the following discussion, when we talk about the redundancy of a relation $P$ in a (or reduced) presentation $(S, \mathcal{P})$ (or $\left(S, \mathcal{P}^{\text {re }}\right)$ ) of $G$, we often simply state " $P$ is not redundant " for " $P$ is not redundant in $(S, \mathcal{P})\left(\right.$ or $\left.\left(S, \mathcal{P}^{\text {re }}\right)\right)$ " if $(S, \mathcal{P})$ (or $\left.\left(S, \mathcal{P}^{\text {re }}\right)\right)$ is clear in the context. By Lemma $7.5(3)$, 
when a relation $P$ is in $\mathcal{P}^{\text {re }}$, to show that $P$ is not redundant in $\mathcal{P}^{\text {re }}$, we need only show that $P$ is not redundant in $\mathcal{P}$ (we always do in this way in the subsequent discussion).

7.8. Let us first consider the group $G_{24}$. We can show that the generators $s_{1}, s_{2}$ are symmetric for the presentation of $G_{24}$ in Proposition 4.3 in the following sense: suppose that relations $\left(\mathrm{a} i^{\prime}\right), 1 \leqslant i \leqslant 3$, are obtained from (ai) by interchanging $s_{1}, s_{2}$, then two relation sets (a1)-(a3) and $\left(\mathrm{a} 1^{\prime}\right)-\left(\mathrm{a} 3^{\prime}\right)$ can be obtained from one to another. We can also show that $s_{1}, s_{3}$ (resp. $\left.s_{1}, s_{3}, s_{8}\right)$ are symmetric for the presentation of $G_{24}$ in Proposition 4.4 (resp. Proposition 4.5). Since the reflection group $G_{24}$ is complex, relations (a5), (b5) are not redundant by Lemma 7.5 (1), and neither are relations (a4) and (b4) by the proof of Proposition 4.4 and by Lemma 7.6. By symmetry, relations (a2) and (b3) are not redundant. Relations (a2), (b2) and (c2) are the same. So neither of (b2) and (c2) is redundant by the proof of Propositions 4.4, 4.5 and by Lemma 7.6. Again by the proof of Proposition 4.5 and by Lemma 7.6, relation (c5) is not redundant. By the symmetry of $s_{1}, s_{8}$ for the presentation of $G_{24}$ in Proposition 4.5, neither of relations (c3) and (c4) is redundant. Finally, by the proof of Proposition 4.5 and by Lemma 7.6, relation (a3) is not redundant. This proves the essentiality of the reduced forms for all the presentations of $G_{24}$ in Propositions 4.3, 4.4 and 4.5.

7.9. Now we consider the group $G_{25}$. We claim that relation (a2) is not redundant. For, relation $s_{1} s_{2}=s_{2} s_{1}$ together with relations (a1), (a3) and (a4) determine a reducible reflection group $\left\langle s_{1}\right\rangle \times\left\langle s_{2}, s_{3}\right\rangle$, meaning that (a2) is not a consequence of relations (a1), (a3), (a4). Similarly, (a3) is not redundant. We claim that relation (a4) is not redundant. For, by replacing $s_{6}$ by $s_{3}$ for the presentation of $G_{25}$ in Proposition 5.4, relations (b1)-(b3) becomes (a1)-(a3), respectively, and (b5) becomes $s_{1} s_{3} s_{1}=s_{3} s_{1} s_{3}$, differing from (a4), which shows that relation (a4) is not a consequence of relations (a1)-(a3). So the reduced form for the presentation of $G_{25}$ in Proposition 5.3 is essential. Then by the proof of Proposition 5.4 and by Lemma 7.6, we see that neither of relations (b2) and (b4) is redundant. Then by the symmetry of $s_{1}, s_{2}, s_{6}$ for the presentation of $G_{25}$ in Proposition 5.4, we see that relation (b3) is not redundant, too. This implies that the reduced form for the presentation of $G_{25}$ in Proposition 5.4 is essential. Finally, to show that the reduced form for the presentation of $G_{25}$ in Corollary 5.5 is essential, we need only show that relation (b3') is not redundant. But this follows by the symmetry of $s_{1}, s_{2}, s_{6}$ for the presentation of $G_{25}$ in Corollary 5.5. 
The proof is similar for the essentiality of the reduced form for the presentations of the group $G_{26}$ in Propositions 6.3 and 6.4.

7.10. Finally we consider the group $G_{12}$. Relation (a3) is not redundant, since (b1) and (b4) become (a1) and (a2) respectively by replacing $s_{10}$ by $s_{3}$, while (b3) does not become (a6) under the same substitution. By symmetry of $s_{1}, s_{2}, s_{3}$ for the presentation of $G_{12}$ in Proposition 3.3, we see that relation (a2) is not redundant, too. So the reduced form for the presentation of $G_{12}$ in Proposition 3.3 is essential. By Lemma 7.5 (1), relation (b4) is not redundant. We claim that relation (b3) is not redundant. For, relation $s_{1} s_{10} s_{1} s_{10} s_{1} s_{10}=$ $s_{10} s_{1} s_{10} s_{1} s_{10} s_{1}$ does not conflict with (b1), (b2) and (b4) by observing the presentation of $G_{12}$ in Proposition 3.3 with $s_{3}$ in the place of $s_{10}$. Next we claim that relation (b2) is not redundant. To see this, one need only notice that with respect to the presentation of $G_{12}$ in Proposition 3.3, the images $\bar{s}_{1}, \bar{s}_{2}, \bar{s}_{3}$ of the generators $s_{1}, s_{2}, s_{3}$ in the quotient group of $G_{12}$ over its center $Z$ ( $Z$ has order two) satisfy relations (b1), (b3), (b4) with $\bar{s}_{1}, \bar{s}_{2}, \bar{s}_{3}$ in the places of $s_{1}, s_{2}, s_{10}$ respectively, and relation $\bar{s}_{1} \bar{s}_{2} \bar{s}_{1}=\bar{s}_{2} \bar{s}_{1} \bar{s}_{2}$. Hence the reduced form for the presentation of $G_{12}$ in Proposition 3.4 is essential. The non-redundancy of relation (c3) follows by the proof of Proposition 3.5 and by Lemma 7.6. Then by the symmetry of $s_{1}, s_{7}$ for the presentation of $G_{12}$ in Proposition 3.5, we see that relation (c2) is not redundant, too. This implies that the reduced form for the presentation of $G_{12}$ in Proposition 3.5 is essential. By the proof of Proposition 3.6 and by Lemma 7.6, we see that neither of relations (d2) and (d3) is redundant and hence the reduced form for the presentation of $G_{12}$ in Proposition 3.6 is essential. Finally consider the presentation of $G_{12}$ in Proposition 3.7. Relation (e5) is not redundant by Lemma 7.5 (1). Relation (e2) is not redundant since one gets a presentation of the quotient group $G_{12} / Z$ (isomorphic to the symmetric group $S_{4}$ ) by replacing (e2) by $s_{1} s_{7}=s_{7} s_{1}$ in Proposition 3.7. Next we claim that relation (e3) is not redundant. For, substituting $s_{1}, s_{7}, s_{8}$ by $s_{2}, s_{8}, s_{1}$ in relations (e1)-(e5) respectively, followed by replacing a resulting relation $s_{1} s_{2} s_{1}=s_{2} s_{1} s_{2}$ (which comes from (e3)) by (d6), all the relations we get are the consequence of the presentation of $G_{12}$ in Proposition 3.6. This shows the claim. By symmetry of $s_{1}, s_{7}$ in the presentation, we see that relation (e4) is not redundant, too. So the reduced form for the presentation of $G_{12}$ in Proposition 3.7 is essential.

\section{Appendix.}

Here we record the results of L. Wang and P. Zeng on the numbers $N(G)$ of inequivalent 
simple root systems for the primitive complex reflection groups $G_{7}, G_{11}, G_{13}, G_{15}, G_{19}$, $G_{22}, G_{27}, G_{29}, G_{31}, G_{32}, G_{33}$.

\begin{tabular}{|c|c|c|c|}
\hline$G$ & $N(G)$ & $G$ & $N(G)$ \\
\hline$G_{7}$ & 2 & $G_{11}$ & 4 \\
\hline$G_{13}$ & 4 & $G_{15}$ & 4 \\
\hline$G_{19}$ & 6 & $G_{22}$ & 18 \\
\hline$G_{27}$ & 6 & $G_{29}$ & 9 \\
\hline$G_{31}$ & 61 & $G_{32}$ & 5 \\
\hline$G_{33}$ & 14 & & \\
\hline
\end{tabular}

Since any complex reflection group generated by one or two reflections has a unique inequivalent simple root system, so far $G_{34}$ is the only group left unknown concerning the inequivalent simple root systems.

\section{REFERENCES}

1. M. Broue, G. Malle and R. Rouquier, Complex reflection groups, Braid groups, Hecke algebras, J. Reine Angew. Math. 500 (1998), 127-190.

2. Arjeh M. Cohen, Finite complex reflection groups, Ann. scient. Éc. Norm. Sup. $4^{e}$ série t. 9 (1976), 379-436.

3. R. B. Howlett and J. Y. Shi, On regularity of finite reflection groups, manuscripta mathematica 102(3) (2000), 325-333.

4. D. W. Koster, Complex reflection groups, Ph.D. thesis, University of Wisconsin (1975).

5. G. C. Shephard and J. A. Todd, Finite unitary reflection groups, Canad. J. Math. 6 (1954), 274-304.

6. J. Y. Shi, Certain imprimitive reflection groups and their generic versions, Trans. Amer. Math. Soc. 354 (2002), 2115-2129.

7. V. L. Popov, Discrete complex reflection groups, Communications of the Mathematical Institute, Rijksuniversiteit Utrecht 15. (1982), 1-89.

8. Li Wang, Simple root systems and some presentations for the primitive reflection groups generated by reflections of order 2, Master Dissertation, East China Normal University, 2003.

9. Peng Zeng, Simple root systems and presentations for the primitive reflection groups containing a reflection of order $>$ 2, Master Dissertation, East China Normal University, 2003.

Email: jyshi@math.ecnu.edu.cn 